\title{
COMMENT Save the kidneys in COVID-19
}

\author{
Philip P. Knight ${ }^{1}$ and Akash Deep ${ }^{1}$ \\ Pediatric Research (2021) 90:253-255; https://doi.org/10.1038/s41390-020-01280-x
}

We would like to take the opportunity to thank Kache et al. for their insightful review of the current therapeutic recommendations for COVID-19 on PICU. These are the first detailed set of guidelines designed for clinicians both in the high- and limitedresource settings. ${ }^{1}$ COVID-19 in critically sick children is a systemic disease that tends to involve multiple organs, including lungs, heart, liver, kidney and brain. This is postulated to be due to Severe Acute Respiratory Syndrome Coronavirus 2 (SARS-CoV-2) binding to angiotensin-converting enzyme II (ACE2) receptors in infected cells expressed in those organs and may affect the coagulation system. ${ }^{2}$ These guidelines generated 44 recommendations related to pediatric COVID-19 patients presenting with respiratory distress or failure, sepsis or septic shock, cardiopulmonary arrest, hyper-inflammatory phenotype and those requiring adjuvant therapies or ECMO.

In response to this manuscript, we would like to highlight that acute kidney injury (AKI) and kidney replacement therapy (KRT) are evolving areas of the emerging COVID 19 data that we think are not fully addressed in the article. These areas will also have particular relevance to countries with scarce resources. We will demonstrate the wide variation in the incidence of $A K I$ and KRT requirements reported in adult patients with COVID-19 and that it is potentially much higher than initially thought. This has corresponded with our local experience of higher KRT requirements in the adults with COVID-19 at King's College Hospital, London. The adult COVID-19 surge not only put a direct demand on pediatric services through reappropriation of pediatric critical care staff, equipment and space, it also consumed resources particularly effecting availability of KRT machines and consumables. This crisis could be reproduced elsewhere as the pandemic spreads and is a potentially unrecognized burden in pediatric critical care worldwide. These requirements of ongoing and future surges may require novel application of KRT to preserve existing and future KRT resources. Hence, knowledge of AKI in COVID-affected children and principles of management of AKI (diagnosis, prevention and treatment (nondialytic and dialytic)) should form a part of guidelines for the multisystem management of COVID-19-affected children.

We recognize the authors' review of the literature to show AKI as an uncommon sequelae as reported by Wang et al. in their series of 116 patients $^{3}$ and there to be no specific data on the incidence of AKI in critically ill children with COVID-19. Current information on kidney involvement in patients affected by COVID-19 is scarce. This is an evolving situation; as the pandemic spreads and cases of COVID-19 rise, we are able to gather more information. The first initial report from China indeed showed the incidence of AKI in infected adults to be around $0.5 \%$ and utilization of KRT was $0.8 \%{ }^{4}$ There has since been a wide range of reported AKI $(0.5-19 \%)$ and KRT requirements $(0.8-9 \%)$ in COVID-19 patients. The majority of these patients are with normal kidney function at baseline and no pre-existing chronic kidney disease. ${ }^{5}$ We concede these rates of AKI are significantly lower than are reported in the general ICU population which exceeds $40 \%{ }^{6}$ However, with global cases continuing to rise, we believe this to be uncovering a hidden burden on providing KRT to non-COVID adult and pediatric patients that needs to be factored into future planning. In pediatrics, the clinical course and treatment of these patients have been similar to adults, though under $1 \%$ have been critically unwell with severe symptoms. ${ }^{7}$ In the multicenter cohort study of children with severe or critically unwell with COVID-19, the Critical Coronavirus And Kids Epidemiologic study (CAKE study), AKI occurred in $18 \%$ of patients. ${ }^{8}$ The study does not mention about the requirement for AKI.

Compounding this is the new phenomenon affecting previously asymptomatic children with SARS-CoV2 infection manifesting as a hyper-inflammatory syndrome with multiorgan involvement. This syndrome has been named Paediatric Inflammatory Multisystem Syndrome temporally associated with SARS-CoV2 (PIMS-TS) in the UK. In a single-center study of 52 patients with COVID-19, 28.8\% developed AKI $(n=15) ; 24$ patients of this cohort had PIMS-TS, of which 14 developed AKI-incidence of $58.3 \%$. In other words, $93.3 \%$ of the patients who developed AKI had a hyper-inflammatory condition. ${ }^{9}$

With over a hundred pediatric patients reported to have presented with a similar clinical syndrome in New York (coined Multisystem Inflammatory Syndrome Associated with COVID-19 (MIS-C) in the US) ${ }^{10}$ this has the potential to put further strain on critical care provision and any increase in demands in pediatric KRT during a COVID-19 surge may be difficult to meet. Having a lower threshold of diagnosing AKI (though other diagnostic criteria exist, KDIGO definition used), strict monitoring of fluid balance, judicious use of fluids in this condition and avoiding or appropriate dosing of nephrotoxic drugs are some of the standard interventions recommended for this patient group. Whether the use of anti-inflammatory and immune-modulatory drugs will have any benefit on the incidence of AKI still needs to be established.

Although the current KRT requirements of children with COVID19 or as a result of a post-inflammatory process remain low, there are growing reports outlined above that suggest the initial figures are an underestimation. Our local experience is that adult critical care demands will put significant strains on KRT resources. The full effects of COVID- 19 are still being characterized. As part of that surveillance we recommend close monitoring of the incidence of AKI and its associated KRT requirements. Though it is extremely difficult to recommend COVID-specific guidelines for the management of AKI, surveillance to diagnose and prevent $\mathrm{AKI}$ is paramount. These are unprecedented circumstances that no one expected, we were all forced to adapt to the new normal and do things which we would not have done had the number of patients not being astronomically high.

\footnotetext{
${ }^{1}$ Pediatric Intensive Care Unit, King's College Hospital, London, UK

Correspondence: Akash Deep (akash.deep@nhs.net)
}

Received: 28 July 2020 Revised: 17 September 2020 Accepted: 23 October 2020

Published online: 19 November 2020 
Diagnose AKI using KDIGO criteria. Treat anticipated risk factors, e.g., assessment of fluid status, treat hypovolemia and shock, prevent fluid overload by judiicious use of diuretics, avoid nephrotoxic medications

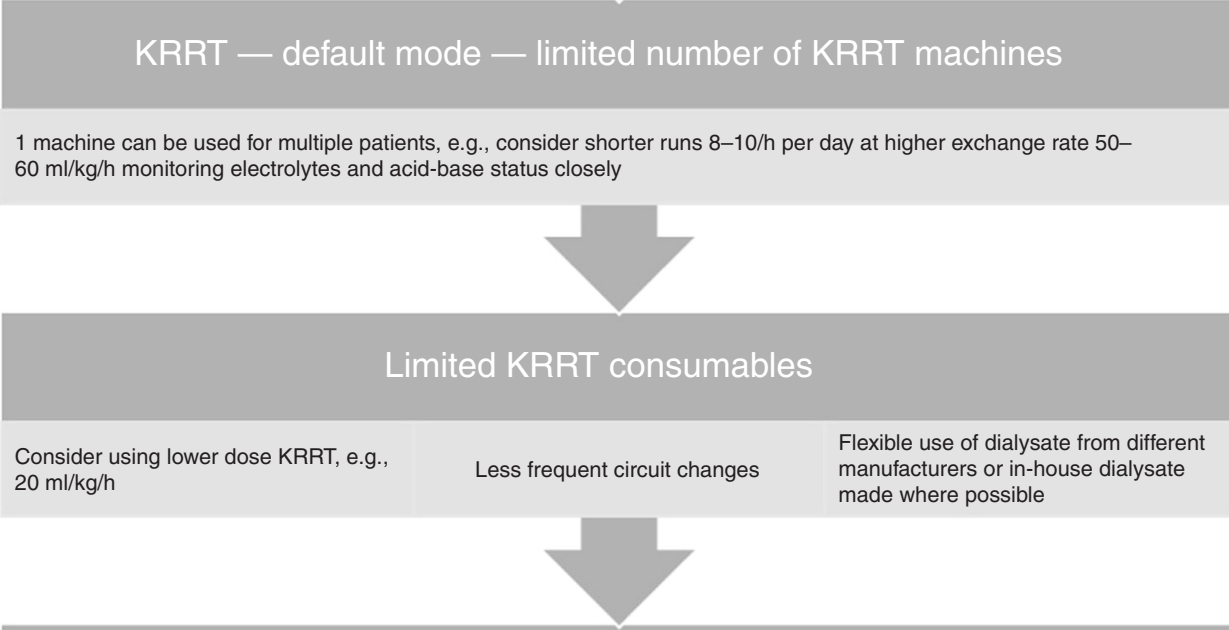

\section{Anticoagulation: frequently clotting filters}
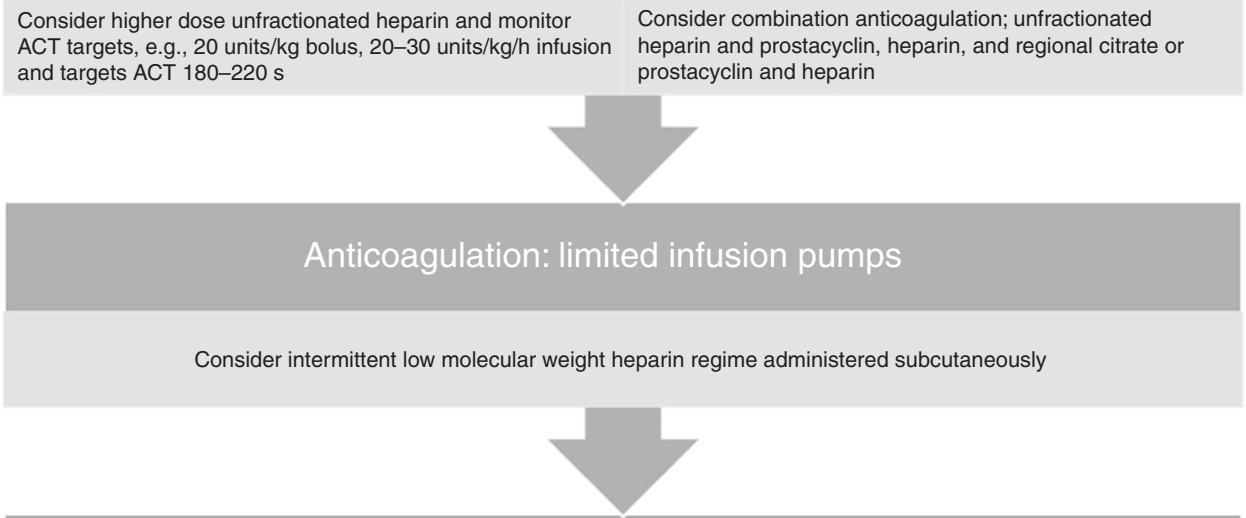

\begin{tabular}{lll} 
& Alternative KRT methods \\
\hline Peritoneal dialysis & $\begin{array}{l}\text { IHD in hemodynamically stable } \\
\text { patients }\end{array}$ & $\begin{array}{l}\text { Routine use of hemadsorption not } \\
\text { recommended. Center-specific use in } \\
\text { the context of research or trials }\end{array}$
\end{tabular}

ACT - activated clotting time; AKI - acute kidney injury; CKRT-continuous kidney replacement Therapy; IHD — intermittent hemodialysis; KRT — kidney replacement therapy.

Fig. 1 Summary of acute kidney Injury and kidney replacement therapy in the context of COVID-19 and limited resources.

There needs to be careful allocation and usage of KRT consumables and machines in future COVID-19 and winter surges. We have outlined potential strategies to preserve resources that include situations where KRT machines are limited, consumables are limited or frequently consumed by circuit clotting (Fig. 1). Most of these recommendations in the table and flowchart are based on experience in our hospital along with other hospitals in London and other countries where resources were exhausted due to unprecedented demand on KRT. American Society of Nephrology (ASN) and pCRRT foundation have recently published recommendations but again these are not evidence based but based on practicalities, common sense and availability of resources. ${ }^{11,12}$

As described by the authors in the section on adjuvant therapy, these patients are hypercoagulable especially the ones with hyper-inflammatory phenotype. ${ }^{13}$ These patients are at risk of frequent filter clotting, which will increase the treatment downtimes, decrease the efficacy of treatment and increase the exposure to healthcare professionals. Therefore, vigilance in the use of circuit, patient and anti-coagulant-related factors to maintain the patency of the continuous kidney replacement 
Table 1. Best practice recommendations for prescription of continuous kidney replacement therapy (CKRT) in COVID-19 patients based on the experience in different hospitals in London and The American Society of Nephrology (ASN) and pCRRT foundation recommendations. ${ }^{11,} 12$

1. Indication: fluid overload, azotemia, electrolyte imbalance (CKRT might have to be initiated at a later stage of AKI rather than early)

2. Reduce exposure to healthcare professionals: To avoid exposure to healthcare staff, CRRT machine can be set outside the isolation room using extended tubing (risk of more frequent disconnections, more priming volume required, hence might not be a feasible option in children)

3. Timing of initiation: There is no evidence to initiate RRT early, especially when resources are limited, STARRT AKI study refutes the advantage of accelerated versus standard timing of initiation of CKRT in critically ill adults

4. Vascular access: largest possible catheter in the right internal jugular vein under ultrasound guidance (best site to access when patient is in the prone position for respiratory failure, catheter-related issues like bending, kinking can also be avoided in this site)

5. Priming solution: Blood, saline, albumin depending on local policy

6. Blood flow rate: based on weight and age of the child as per standard non-COVID guidelines, might have to be increased to prevent excessive filter clotting

7. Filter size: as per size of the patient, might have to increase the size to prevent thrombosis.

8. Circuit and blood line set: as standard, depending on resources, we might have to adapt to 'whatever' is available and safe

9. Dialysate/Replacement fluid dose: If fluids and CKRT machines are available-use standard recommended dose; if fluids are in short supply, use lesser exchange rates for $24 \mathrm{~h}$. If fluids in extreme shortage-fluids from different machines can be interchangeably used. Some centers manufacture their own dialysis fluid

10. Net ultrafiltration rate: $1-2 \mathrm{ml} / \mathrm{kg} / \mathrm{h}$ after the patient is haemodynamically stable

11. Anticoagulation: most important aspect of CKRT in COVID-19 due to frequent filter clotting due to hyper-coagulable state-unfractionated heparin (UFH), regional citrate anticoagulation (RCA), prostacyclin, low molecular weight heparin (LMWH) most commonly used either systemically, regionally or combination of the 2 . If filter life still low, a combination of anticoagulants can be used (UFH + RCA/ UFH + citrate/LMWH + RCA)

12. Stopping CRRT: same principles as used for non-COVID, might have to change criteria to give filter holiday to spare the machines for other patients who could not be offered CRRT

13. Drug dose alteration: standard principles to follow; information on drugs used on CKRT in COVID-19 patients—ramdesevir, tocilizumab, not yet available

therapy (CKRT) circuit is paramount in these patients. Use of alternate modalities of KRT in the ICU population (peritoneal dialysis and intermittent hemodialysis) during the pandemic could decrease dependence on CKRT machines especially in the resource-crisis scenario. These strategies could be considered early in surges and prior knowledge of this, especially in resource-limited settings, could make the difference at peak times of being able to provide KRT and leaving patients unable to receive this support. There is no evidence to support the recommendations to initiate, maintain or terminate CKRT even in patients with non-COVID etiologies. Based on worldwide experience in managing KRT during the pandemic, and our own experience at King's College Hospital, London, we have summarized our best practice suggestions for prescribing CKRT in the setting of COVID-19 (Table 1). Lastly, the use of cytokine removing extra-corporeal modalities in the form of haemoperfusion, medium- to high cut-off membranes and using tandem therapies are still being used under research/experimental umbrella. Therefore, it is vital that we create awareness to actively look out for AKI, diagnose and prevent AKI pro-actively and have contingency plans in place to initiate KRT in cases that fulfill the criteria to receive KRT. We thank the authors again for their valuable initiative in this rapidly changing global crisis.

\section{DISCLAIMER}

We declare that the work is original, not previously published, and not submitted for publication or consideration elsewhere.

\section{AUTHOR CONTRIBUTIONS}

P.P.K. was responsible for contributions to design, acquisition of data, or analysis and interpretation of data and drafting the article. A.D. was responsible for concept, revising it critically for important intellectual content and final approval of the version submitted for publication.

\section{ADDITIONAL INFORMATION}

Competing interests: The authors declare no competing interests.
Publisher's note Springer Nature remains neutral with regard to jurisdictional claims in published maps and institutional affiliations.

\section{REFERENCES}

1. Kache, S. et al. COVID-19 PICU guidelines: for high- and limited-resource settings. Pediatr. Res. https://doi.org/10.1038/s41390-020-1053-9 (2020).

2. Li, S.-R., Tang, Z.-J., Li, Z.-H. \& Liu, X. Searching therapeutic strategy of new coronavirus pneumonia from angiotensin-converting enzyme 2: the target of COVID-19 and SARS-CoV. Eur. J. Clin. Microbiol. Infect. Dis. 39, 1021-1026 (2020).

3. Wang, L. et al. Coronavirus disease 19 infection does not result in acute kidney injury: an analysis of 116 hospitalized patients from Wuhan, China. Am. J. Nephrol. 51, 343-348 (2020).

4. Guan, W. J. et al. China Medical Treatment Expert Group for Covid-19. Clinical characteristics of coronavirus disease 2019 in China. N. Engl. J. Med. https://doi. org/10.1056/NEJMoa2002032 (2020).

5. Zaim, S., Chong, J. H., Sankaranarayanan, V. \& Harky, A. COVID-19 and multiorgan response. Curr. Probl. Cardiol. 45, 100618 (2020).

6. Hoste, E. A. et al. Epidemiology of acute kidney injury in critically ill patients: the multinational AKI-EPI study. Intensive Care Med. 41, 1411-1423 (2015).

7. Dong, Y. et al. Epidemiology of COVID-19 among children in China. Pediatrics https://doi.org/10.1542/peds.2020-0702 (2020).

8. González-Dambrauskas, S. et al. Critical Coronavirus and Kids Epidemiology (CAKE) Study. Pediatric critical care and COVID19. Pediatrics https://doi.org/ 10.1542/peds.2020-1766 (2020).

9. Stewart, D. J. et al. Kidney dysfunction in hospitalised children with COVID-19 [published online ahead of print, 2020 Jun 15]. Lancet Child Adolesc. Health S2352-4642, 30178-4 (2020).

10. Centers for Disease Control and Prevention Health Alert Network (HAN). Multisystem Inflammatory Syndrome in Children (MIS-C) Associated with Coronavirus Disease 2019 (COVID-19). 2020; Available at: https://emergency.cdc.gov/han/ 2020/han00432.asp. (Accessed on May 15, 2020).

11. American Society of Nephrology. Recommendations on the care of hospitalized patients with COVID-19 and kidney failure requiring Renal Replacement Therapy. [cited 2020 April 13]. Available from: https://www.asn-online.org/covid.19/ ASN\#ASN_Recommendations 2020.

12. Raina, R., Chakraborty, R., Sethi, S. K. \& Bunchman, T. Kidney replacement therapy in COVID-19 induced kidney failure and septic shock: a pediatric continuous renal replacement therapy [PCRRT] position on emergency preparedness with resource allocation. Front. Pediatr. 8, 413 (2020).

13. Connors, J. M. \& Levy, J. H. COVID-19 and its implications for thrombosis and anticoagulation. Blood 135, 2033-2040 (2020). 